\title{
Rearrangement of Sodium ordering and its effect on physical properties in $\mathrm{Na}_{x} \mathrm{CoO}_{2}$ system
}

\author{
T. Wu, K. Liu, H. Chen, G. Wu, Q. L. Luo, J. J. Ying and X. H. Chen® \\ Hefei National Laboratory for Physical Science a Microscale and Department of Physics, \\ University of Science and Technology of China, Hefei, \\ Anhui 230026, People's Republic of China
}

\begin{abstract}
We systematically study Raman spectroscopy of cleaved $\mathrm{Na}_{x} \mathrm{CoO}_{2}$ single crystals with $0.37 \leq \mathrm{x}$ $\leq 0.80$. The Raman shift of $\mathrm{A}_{1 g}$ mode is found to be linearly dependent on Na content, while the Raman shift of $\mathrm{E}_{1 g}$ mode has an abnormal shift to high frequency around $\mathrm{x}=0.5$. The abnormal shift is ascribed to the occurrence of $\mathrm{Na}$ rearrangement in $\mathrm{O} 1$ structure. Temperature dependent Raman spectrum for $\mathrm{x}=0.56$ sample shows that $\mathrm{Na}$ rearrangement transition from $\mathrm{O} 1$ structure to $\mathrm{H} 1$ structure occurs around $240 \mathrm{~K}$. Electronic transport and susceptibility for the sample with $x=0.56$ show a response to the Na rearrangement transition from $\mathrm{O} 1$ to $\mathrm{H} 1$ structure, and that different $\mathrm{Na}$ ordering pattern causes distinct physical properties. These results give a direct evidence to proved $\mathrm{Na}$ ordering effect on physical properties of $\mathrm{Co}-\mathrm{O}$ plane.
\end{abstract}

PACS numbers: 31.30.Gs,71.38.-k,75.30.-m

\section{INTRODUCTION}

The layered cobaltate $\mathrm{Na}_{x} \mathrm{CoO}_{2}$ has attracted much interesting in strong correlation research area due to its rich physics $\frac{1,2,3}{1}$ As we know, the electronic ground state is strongly dependent on the $\mathrm{Na}$ content in $\mathrm{Na}_{x} \mathrm{CoO}_{2} \frac{3}{\text { in }}$ which the valence of $\mathrm{Co}$ ion can be tuned by $\mathrm{Na}$ content. Moreover, the change of $\mathrm{Na}$ content can also affect the structure of $\mathrm{Na}^{+}$ion layer. Early electron diffraction and neutron diffraction measurements reveal a kaleidoscope of $\mathrm{Na}^{+}$ion patterns as a function of concentration ${ }^{4,5,6,7}$ A detailed phase diagram of $\mathrm{Na}^{+}$ion layer structure was given by Huang et al. ${ }^{6}$ As shown in Fig.1, there are four different types of structure for $\mathrm{Na}^{+}$ion layer with different $\mathrm{Na}$ content - $\mathrm{H} 1, \mathrm{H} 2, \mathrm{H} 3$ and $\mathrm{O} 1$. In the four types of structure, there are three types of $\mathrm{Na}^{+}$ion site - $\mathrm{Na}(1)(2 \mathrm{~b}(0,0,1 / 4)), \mathrm{Na}(2)(2 \mathrm{c}(2 / 3,1 / 3,1 / 4))$ and $\mathrm{Na}(2)^{\prime}(6 \mathrm{~h}(2 \mathrm{x}, \mathrm{x}, 1 / 4))$. The occupation probability of the above sites depends on both $\mathrm{Na}$ content and temperature ${ }^{6}=$ It is expected that different $\mathrm{Na}^{+}$ion pattern corresponds to distinct physical properties. The relationship between structure of $\mathrm{Na}^{+}$ion layer and physical properties of Co-O plane is believed to be an important key of understanding this system ${ }^{3,6}$ and becomes a hot issue in recent researches $8,9,10,11,12,13,14,15,16$.

Recently, single crystal neutron scattering study reveals the formation of ordered sodium vacancies for $\mathrm{x}>0.5$. Inside each vacancy, there exists a $\mathrm{Na}$ monomer or a $\mathrm{Na}$ trimer occupying $\mathrm{Na}(1)$ sites that sit atop $\mathrm{Co}$ atoms. $\stackrel{8}{-}$ This multi-vacancy model and $\mathrm{Na}$ trimer geometry are also proved by single crystal synchrotron X-ray diffraction and can be used to explain the observed superstructures of $\mathrm{x}=0.84$ and $\mathrm{x}=0.71$ crystals very well. ${ }^{11}$

*Corresponding author; Electronic address: chenxh@ustc.edu.cn

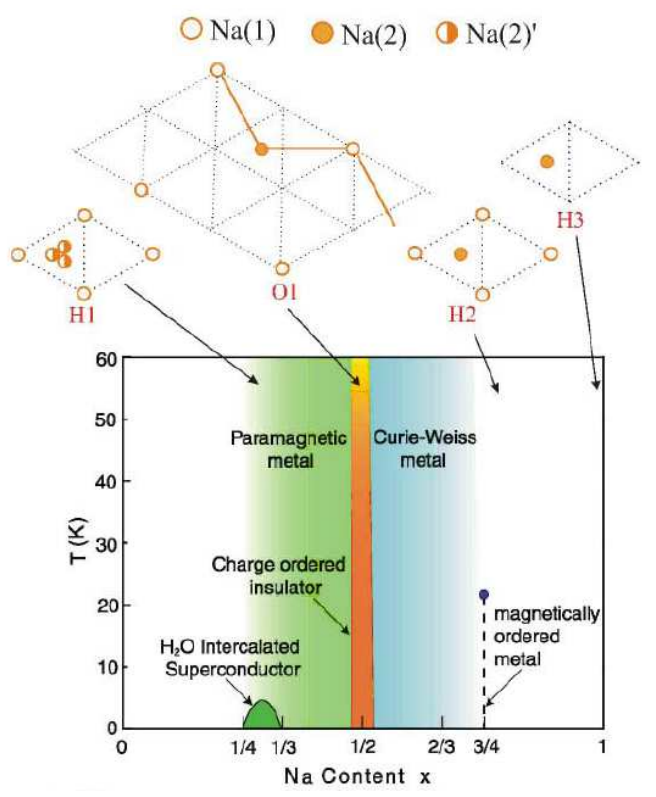

FIG. 1: (Color online). Correspondence between structure and properties in $\mathrm{Na}_{x} \mathrm{CoO}_{2}$ (Figure comes from ref[6]). Upper panel: schematic of the $\mathrm{Na}$ ion distributions in the four $\mathrm{Na}_{x} \mathrm{CoO}_{2}$ phases; Lower panel: the electronic phase diagram.

A recent STM study also found that an unexpected $\mathrm{Na}$ trimer ordering is found for $\mathrm{x} \leq 0.5$ samples $\underline{13}$ These interesting findings make us renew our understanding of $\mathrm{Na}$ ordering pattern. More and more theoretical and experimental progress of $\mathrm{Na}$ ordering $8,11,14,15,16$ show that $\mathrm{Na}$ ordering plays an important role in understanding of the rich physical properties of $\mathrm{Co}-\mathrm{O}$ plane. However, direct experimental evidence of relationship between $\mathrm{Na}$ ordering and physical properties of Co-O plane is still limited ${ }^{9}$. Here, we report a systematically Raman study in $\mathrm{Na}_{x} \mathrm{CoO}_{2}$. It is found that an abnormal sodium depen- 
dence of $\mathrm{E}_{1 g}$ mode occurs around $\mathrm{x}=0.5$. Temperature dependent Raman study indicates that a possible Na rearrangement transition from $\mathrm{H} 1$ to $\mathrm{O} 1$ phase defined by Huang et $a l \underline{\underline{6}}$ occurs at $\mathrm{x}=0.56$. Electronic transport and susceptibility for the sample with $\mathrm{x}=0.56$ show that this kind of $\mathrm{Na}$ rearrangement transition makes different physical properties in this system. These results give a direct evidence for $\mathrm{Na}$ ordering effect on the physical properties of Co-O plane.

\section{EXPERIMENT}

High quality single crystals of $\mathrm{Na}_{x} \mathrm{CoO}_{2}$ were grown using the flux method $(\mathrm{x}=0.7)$ and floating zone technique $(\mathrm{x}=0.75$ and 0.80$)$. The $\mathrm{Na}_{x} \mathrm{CoO}_{2}$ sample with $\mathrm{x}<0.7$ is prepared by sodium deintercalation of the $\mathrm{Na}_{0.7} \mathrm{CoO}_{2}$ singe crystals in solutions obtained by dissolving $\mathrm{I}_{2}(0.2$ $\mathrm{M}, 0.02 \mathrm{M}, 0.004 \mathrm{M})$ or $\mathrm{Br}_{2}(6.0 \mathrm{M})$ in acetonitrile $(\mathrm{M}$ is "molar") for 4 days at room temperature. The $\mathrm{x}$ values of the samples were estimated by the same method as previous paper $\stackrel{17}{=}$ Raman spectra were obtained on a LABRAM-HR Confocal Laser MicroRaman Spectrometer using the $514.5 \mathrm{~nm}$ line from an argon-ion laser with in-plane light polarization. The single crystals were cleaved to obtain fresh surface before Raman measurements. The resistance was measured by an $\mathrm{AC}$ resistance bridge (LR-700, Linear Research). Magnetic susceptibility measurements were performed with a superconducting quantum interference device magnetometer in a magnetic field of $7 \mathrm{~T}$. It should be addressed that all results discussed as follow are well reproducible.

\section{RESULT AND DISCUSSION}

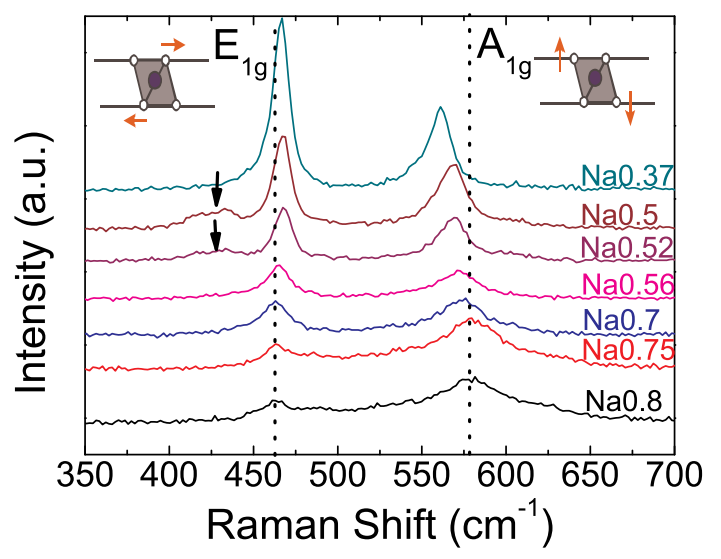

FIG. 2: (color online). Raman spectra of $\mathrm{Na}_{x} \mathrm{CoO}_{2}$ single crystal from ab-plane with $\mathrm{x}=0.37,0.50,0.52,0.56,0.70,0.75$, 0.80 at room temperature.

Fig. 2 shows the Raman spectra for $\mathrm{x}=0.37,0.50,0.52$, $0.56,0.7,0.75$ and 0.80 at ambient temperature with the range from 350 to $700 \mathrm{~cm}^{-1}$. Raman spectra of all samples are measured from ab-plane with in-plane light polarization. In Fig.2, all samples show two distinct Raman modes around $467 \mathrm{~cm}^{-1}$ and $561 \mathrm{~cm}^{-1}$, which can be attributed to Raman-active in-plane $\mathrm{E}_{1 g}$ and out-of-plane $\mathrm{A}_{1 g}$ vibrations of oxygen in $\mathrm{CoO}_{6}$ octahedra, respectively. $\frac{18,19,20,21,22,23}{2}$ The samples with $\mathrm{x}=$ 0.5 and 0.52 show additional modes around $425 \mathrm{~cm}^{-1}$, which is attributed to $\mathrm{Na}^{+}$ion ordering $\underline{21}$ In addition, both of two samples show a charge ordering behavior at low temperature which is consistent with the results by Foo et al $\stackrel{3}{\underline{n}}$ and $\mathrm{Wu}$ et al. $\frac{17}{}$ These results indicate the sensitivity of this phonon to structural and electronic ordering processes. Two distinct systematic shifts are observed for $\mathrm{A}_{1 g}$ and $\mathrm{E}_{1 g}$ modes. With decreasing $\mathrm{Na}$ content, the $\mathrm{A}_{1 g}$ mode shifts to low frequency and $\mathrm{E}_{1 g}$ mode shifts to high frequency. The widths of $\mathrm{A}_{1 g}$ and $\mathrm{E}_{1 g}$ modes increase with sodium doping, and there exists a distinct broadening of $\mathrm{A}_{1 g}$ and $\mathrm{E}_{1 g}$ modes above $\mathrm{x} \sim 0.52$. This broadening of the two modes maybe come from instability of ordering structure of $\mathrm{Na}^{+}$ions. The systematic changes of related Raman shift of $\mathrm{A}_{1 g}$ and $\mathrm{E}_{1 g}$ with decreasing $\mathrm{Na}$ content are shown in Fig.3. The Raman shift of $\mathrm{A}_{1 g}$ mode is linearly suppressed with $\mathrm{Na}$ content. This result is consistent with previous data $.23,24$ But it is surprising that the Raman shift of $\mathrm{E}_{1 g}$ mode presents an abnormal shift around $\mathrm{x}=0.5$, and this phenomenon is not observed in previous data $\stackrel{24}{=}$ Huang et al. have proposed a structural phase diagram based on different structure of $\mathrm{Na}^{+}$ion layer. $\frac{6}{}$ Present data indicate that the $\mathrm{O} 1$ structure may be corresponding to the abnormal shift of $\mathrm{E}_{1 g}$ mode. Meanwhile, above results also indicate that the $\mathrm{E}_{1 g}$ mode is more sensitive to $\mathrm{O} 1$ structure than $\mathrm{A}_{1 g}$ mode, which can be used as the fingerprint of $\mathrm{O} 1$ structure. It should be emphasized that the temperature dependence of Raman shift for $\mathrm{E}_{1 g}$ mode also shows a Tlinear behavior except for that observed in the samples of $x \sim 0.5$ with $\mathrm{Na}$ ordering in O1 structure. Since the in-plane $\mathrm{Na}^{+}$ion ordering happens between two neighboring $\mathrm{CoO}_{6}$ layers, it is also easy to understand why the out-of-plane $\mathrm{A}_{1 g}$ mode has no distinct change. Recently, multi-vacancy model was proposed to understand the $\mathrm{Na}$ ordering phenomenon ${ }^{8}$ and $\mathrm{Na}$ rearrangement phenomena were also found for $\mathrm{x}>0.5 \stackrel{7.10}{\frac{1}{2}}$ The above results suggest that a possible $\mathrm{Na}$ rearrangement transition occurs around $\mathrm{x}=0.5$ and $\mathrm{E}_{1 g}$ mode is a good indicator.

In order to further study the possible Na rearrangement transition, we chose $\mathrm{x}=0.56$ sample and measured temperature dependent Raman shift $(80 \mathrm{~K} \sim 310 \mathrm{~K})$. In Fig.4, temperature dependent Raman spectrum for $\mathrm{x}$ $=0.56$ shows different temperature dependence for $\mathrm{A}_{1 g}$ and $\mathrm{E}_{1 g}$ mode. The temperature dependent Raman shift of $\mathrm{A}_{1 g}$ and $\mathrm{E}_{1 g}$ mode is shown in Fig.4(a). As shown in Fig.4(b) and (c), $\mathrm{A}_{1 g}$ mode linearly changes with decreasing temperature. Although a step-like change happens around $T_{s} \sim 240 \mathrm{~K}$ for the $\mathrm{E}_{1 g}$ mode, Raman shift of the $\mathrm{E}_{1 g}$ mode follows a T-linear dependence below and above $T_{s}$. According to the temperature dependence of Raman 


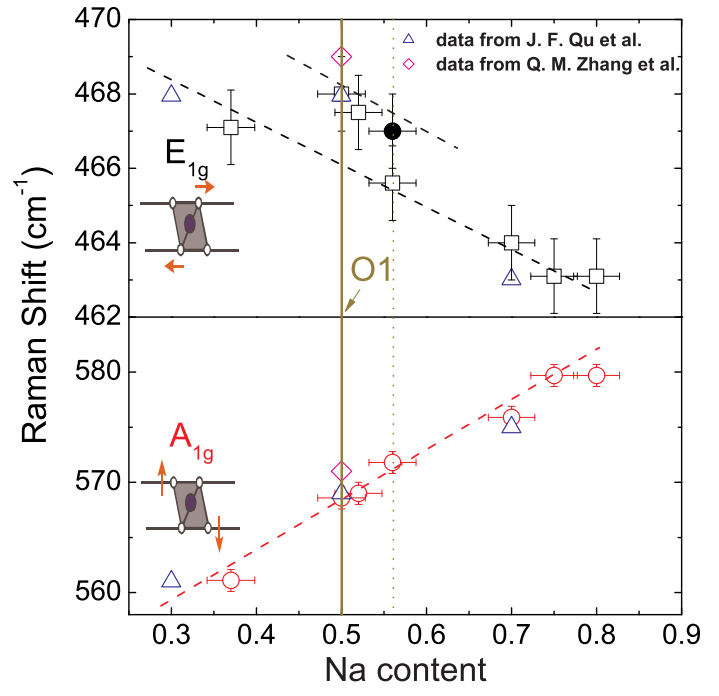

FIG. 3: (color online). Na content dependent Raman shift of $\mathrm{A}_{1 g}$ and $\mathrm{E}_{1 g}$ mode at ambient temperature. Open squares stand for $\mathrm{E}_{1 g}$ mode. Open circles stand for $\mathrm{A}_{1 g}$ mode. Close circle stand for Raman shift of $\mathrm{E}_{1 g}$ mode obtained by extrapolating the temperature dependent Raman shift of $\mathrm{E}_{1 g}$ mode below $\mathrm{Na}$ ordering transition temperature to room temperature for $\mathrm{x}=0.56$ sample. Open triangle and diamond data come from Ref[21] and Ref[22], respectively. Dark yellow line stand for $\mathrm{O} 1$ ordering region phase defined by Huang et al.. Dotted dark yellow line stands for $\mathrm{Na}$ reordering region defined in this paper. The black and red dash line are guided for eyes.

shift below $T_{s}$, a Raman shift could be obtained by extrapolating to room temperature. As shown in Fig.3, the Raman shift of $\mathrm{E}_{1 g}$ mode obtained by extrapolating to room temperature falls to the abnormal shift region around $\mathrm{x}=0.5$ in Na ordering $\mathrm{O} 1$ structure. Since the sample is placed in a airtight box for low temperature measurements, the signal-to-noise ratios of spectra are much lower than those in Fig. 2. We can not see the weak Raman peak around $425 \mathrm{~cm}^{-1}$ below $240 \mathrm{~K}$, which exist in $\mathrm{x}=0.5$ and 0.52 sample. Some previous data are also included in Fig.3, it further indicates a $\mathrm{Na}$ ordering transition from $\mathrm{O} 1$ to $\mathrm{H} 1$ structure at $T_{s} \sim 240 \mathrm{~K}$ with increasing temperature for the sample with $\mathrm{x}=0.56$. Detailed information about this kind of $\mathrm{Na}$ ordering transition needs to confirm by further neutron or electron scattering experiment.

Temperature dependent resistivity and susceptibility for $\mathrm{x}=0.56$ are shown in Fig. 5 in whole temperature region $(2 \mathrm{~K} \leq \mathrm{T} \leq 300 \mathrm{~K})$. In Fig.5(a), the susceptibility under $\mathrm{H}=7 \mathrm{~T}$ parallel to Co-O plane shows an anomaly around $240 \mathrm{~K}$, being consistent with Na reordering transition observed in Raman study. Above $280 \mathrm{~K}$, the temperature dependent susceptibility shows a Curie-Weiss behavior. The data were fitted with the Curie-Weiss law: $\chi=\chi_{0}+\frac{C}{T+\theta}$. The fitting parameter $\theta$ is $124 \mathrm{~K}$ and number of $\mathrm{S}=1 / 2$ local spin is $0.14 /$ Co site. This result is
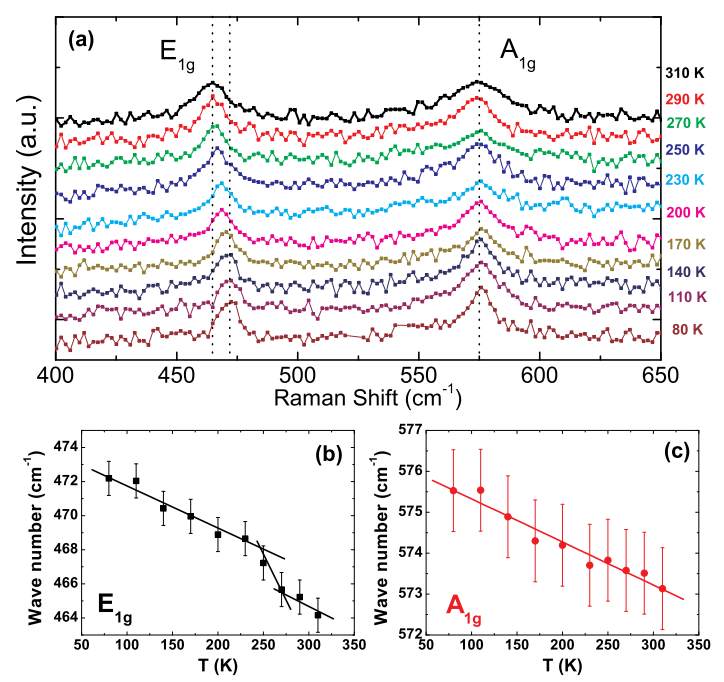

FIG. 4: (color online).(a): Temperature dependent Raman spectra of $\mathrm{Na}_{x} \mathrm{CoO}_{2}$ single crystal with $\mathrm{x}=0.56$; Temperature dependent Raman Shift of (b): $\mathrm{E}_{1 g}$ mode and (c): $\mathrm{A}_{1 g}$ mode for $\mathrm{Na}_{x} \mathrm{CoO}_{2}$ single crystal with $\mathrm{x}=0.56$.

in agreement with the report for $\mathrm{x}=0.7$ sample $\stackrel{\underline{3}}{\text { Below }}$ $240 \mathrm{~K}$, the susceptibility shows a Pauli paramagnetismlike behavior. No antiferromagnetic ordering is observed down to $4 \mathrm{~K}$, unlike $\mathrm{x}=0.5$ sample $^{3}$. A Curie tail exists below $50 \mathrm{~K}$. The Curie tail is fitted with Curie-Weiss law, and the value of $\theta$ is $46 \mathrm{~K}$ and the number of $\mathrm{S}=1 / 2$ local spin is $0.03 /$ Co site. It indicates that the efficient number of $S=1 / 2$ local spin and antiferromagnetic correlation are reduced due to the $\mathrm{Na}$ reordering transition. A crossover from Curie-Weiss to Pauli paramagnetism is accompanied with $\mathrm{Na}$ reordering transition from $\mathrm{H} 1$ to $\mathrm{O} 1$ structure. A linear behavior is observed in field dependent susceptibility at $4 \mathrm{~K}$ and only a tiny nonlinear behavior is observed below $2 \mathrm{~T}$, as shown in inset of Fig.5(a). This also indicates that no ferromagnetic ordering happens, the ferromagnetic ordering shows up in $\mathrm{x}=0.55$ and 0.52 sample $\frac{17.25}{}$ In Fig.5(b), the temperature dependent resistivity shows a loop behavior around $240 \mathrm{~K}$ and the metal behavior is observed in the entire temperature-region. Below $30 \mathrm{~K}$, the temperature dependent resistivity obeys $\mathrm{T}^{1.5}$ law. Between $30 \mathrm{~K}$ and $200 \mathrm{~K}$, a well defined T-linear behavior is found. These characteristics are similar to that in Pauli paramagnetic metal for $\mathrm{x}<0.5$, being consistent with above magnetic properties. Isothermal magnetoresistance and temperature dependent magnetoresistance under $7 \mathrm{~T}$ are shown in Fig.6. A positive and monotonic magnetoresistance is observed as shown in Fig.6(a) and (b). Below $30 \mathrm{~K}$, a prominent positive magnetoresistance is observed under $7 \mathrm{~T}$ with magnetic field both parallel and perpendicular to Co-O plane, which is matched with the $\mathrm{T}^{1.5}$ behavior in resistivity. The maximum of magnetoresistance reaches $24 \%$ and $11 \%$ with $\mathrm{H}$ perpendicular and parallel to Co-O plane, respectively. These results indicate 

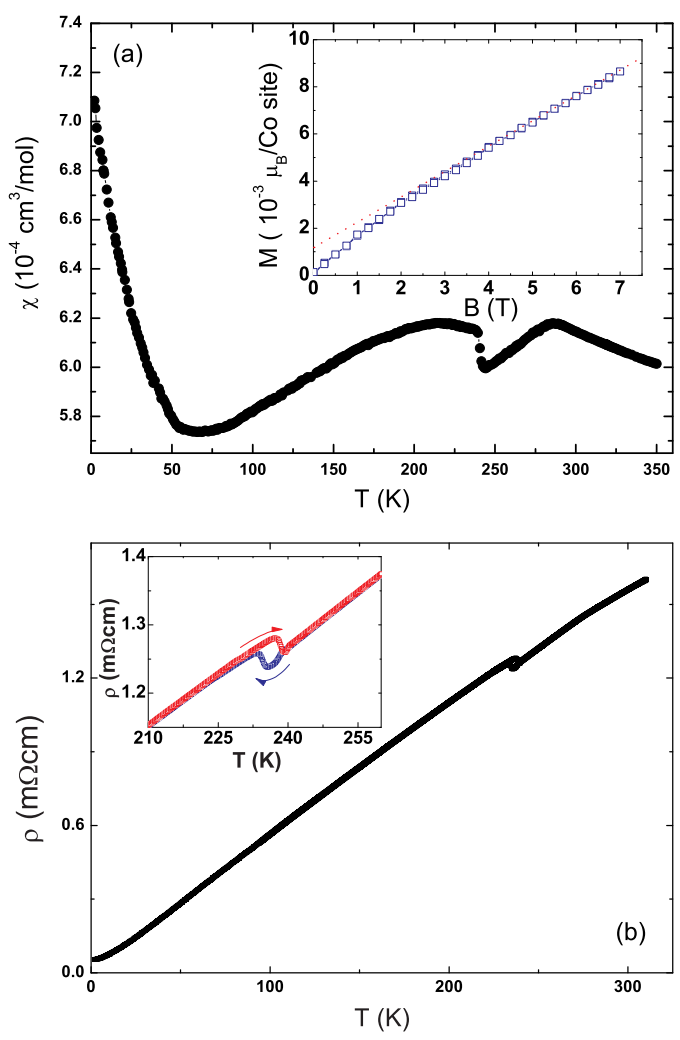

FIG. 5: (color online). Temperature dependent (a): susceptibility with $\mathrm{B}=7 \mathrm{~T}$ and $\mathrm{B} \| \mathrm{Co}-\mathrm{O}$ plane and (b): in-plane resistivity of $\mathrm{Na}_{x} \mathrm{CoO}_{2}$ single crystal with $\mathrm{x}=0.56$. The inset in (a): magnetization as a function of magnetic field up to $7 \mathrm{~T}$ at $4 \mathrm{~K}$. The inset in (b): temperature dependent resistivity around structural transition temperature with cooling and heating measurement.

that strong magnetic fluctuation occurs below $30 \mathrm{~K}$ and a novel paramagnetic state maybe exist at low temperature.

Recently, more and more experiments indicated that $\mathrm{Na}$ ordering is the key to understanding novel properties in $\mathrm{Na}_{x} \mathrm{CoO}_{2}$ system $8,11,14,15,16$ A plenty of $\mathrm{Na}$ ordering pattern and rearrangement transition between these different ordering patterns are found. 10,11 But direct evidence to proved the relationship between $\mathrm{Na}$ ordering pattern and physical properties of $\mathrm{Co}-\mathrm{O}$ plane is still limited $\frac{9}{-}$ The above results give strong evidence that $\mathrm{Na}$ reordering transition from $\mathrm{H} 1$ to $\mathrm{O} 1$ structure can induce a crossover from Curie-Weiss to Pauli paramagnetic metal. Above $\mathrm{Na}$ reordering transition, a CurieWeiss behavior similar to that observed in $\mathrm{x}=0.7$ sample is confirmed by the temperature dependent susceptibility. Below Na reordering transition, temperature dependent resistivity and susceptibility are perfectly consistent with Pauli paramagnetic metal for $\mathrm{x}<0.5$ sample. These results indicate a direct link between $\mathrm{Na}$ reordering transition and crossover from Curie-Weiss to Pauli paramagnetic metal. There exists contradiction about the ab-
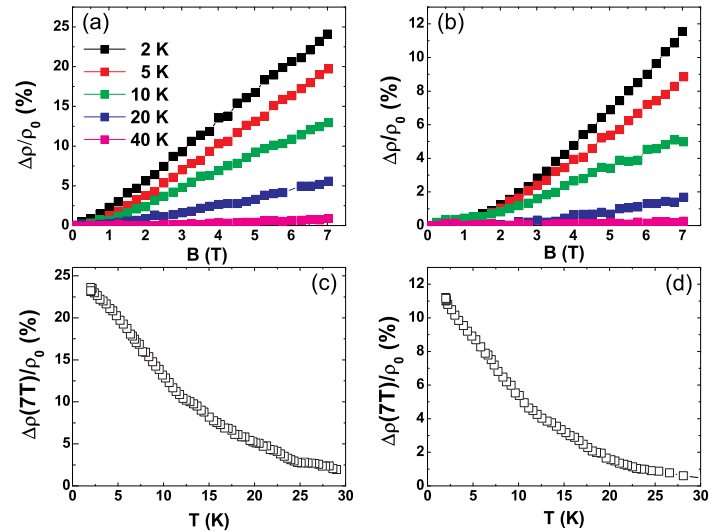

FIG. 6: (color online). Magnetic field dependent in-plane magnetoresistance with external field (a) perpendicular to Co$\mathrm{O}$ plane and (b) parallel to Co-O plane. (c) and (d): magnetoresistance at 7 Tesla as a function of temperature with external field (c) perpendicular to Co-O plane and (d) parallel to Co-O plane.

normal "Curie-Weiss" metal for a long time. Recently, Shubnikov de Haas effect has been studied in $\mathrm{x}=0.84$ and 0.71 samples 12 They found that the $\mathrm{Na}$ ordering can reconstruct the Fermi surface (FS) pockets and leads to the formation of local spin. In this picture, the crossover observed here can be attributed to another kind of reconstruction of FS pockets induced by $\mathrm{Na}$ reordering transition. Local spins are reduced and Pauli paramagnetism is enhanced by this kind of reconstruction of FS pockets. These results also shed light to the mechanism of charge ordering insulator for $\mathrm{x}=0.5$ sample. The charge ordering insulator evolves from Pauli paramagnetic metal. Spin density wave (SDW) or charge density wave(CDW) mechanism is favor for this situation and early NMR result supports this picture. ${ }^{26}$ But many recent experiments show that density wave mechanism can not respond to insulator state for $\mathrm{x}=0.5$ sample, and $\mathrm{Na}$ ordering effect is proposed to understand it. $27,28,29$ The present data can be understood by density wave mechanism, but if there exists any other $\mathrm{Na}$ ordering pattern different from $\mathrm{x}=0.56$ at low temperature same as that in $\mathrm{x}=0.5$, the density wave picture will be challenged.

\section{CONCLUSION}

Raman spectroscopy of cleaved $\mathrm{Na}_{x} \mathrm{CoO}_{2}$ single crystals with $0.37 \leq \mathrm{x} \leq 0.80$ was systematically studied. The Raman shift of $\mathrm{A}_{1 g}$ mode is found to be linearly dependent on Na content. While the Raman shift of $\mathrm{E}_{1 g}$ mode has an abnormal shift to high frequency around $\mathrm{x}=0.5$. The abnormal shift is ascribed to $\mathrm{Na}^{+}$ordering in $\mathrm{O} 1$ structure. Na reordering transition from $\mathrm{O} 1$ to $\mathrm{H} 1$ structure around $240 \mathrm{~K}$ is proved by temperature dependent Raman spectrum for $\mathrm{x}=0.56$ sample. Electronic transport and susceptibility measurement for $\mathrm{x}=0.56$ indicate 
that the Na ordering in different structures leads to distinct physical properties. Present results give a direct evidence for the relationship between $\mathrm{Na}$ ordering and physical properties of $\mathrm{Co}-\mathrm{O}$ plane. Effect of $\mathrm{Na}$ ordering on physical properties is clearly presented

This work is supported by the grant from the Na- ture Science Foundation of China and by the Ministry of Science and Technology of China (973 project No: 2006CB601001 and 2006CB922005), the Knowledge Innovation Project of Chinese Academy of Sciences, and the Innovation Foundation of USTC for the Postgraduate (KD2007077).
1 K. Takada et al., Nature (London) 422, 53(2003).

2 N. P. Ong and R. J. Cava, Science 305, 52(2004).

3 M. L. Foo et al., Phys. Rev. Lett. 92, 247001(2004).

${ }^{4}$ H. W. Zandbergen, M. L. Foo, Q. Xu, V. Kumar and R. J. Cava, Phys. Rev. B 70, 024101(2004).

5 Q. Huang, M. L. Foo, J. W. Lynn, H. W. Zandbergen, G. Lawes, Yayu Wang, B. H. Toby, A. P. Ramirez, N. P. Ong, and R. J. Cava (2004), cond-mat/0402255

${ }^{6}$ Q. Huang, M. L. Foo, R. A. Pascal Jr., J. W. Lynn, B. H. Toby, Tao He, H. W. Zandbergen, and R. J. Cava (2004), cond-mat $/ 0406570$

7 Q. Huang, B. Khaykovich, F.C. Chou, J.H. Cho, J. W. Lynn, Y. S. Lee, Phys. Rev. B 70, 134115(2004).

8 M. Roger, D. J. P. Morris, D. A. Tennant, M. J. Gutmann, J. P. Goff, J.-U. Hoffmann, R. Feyerherm, E. Dudzik, D. Prabhakaran, A. T. Boothroyd, N. Shannon, B. Lake and P. P. Deen, Nature (London) 445 631-634(2007).

9 T. F. Schulze, P. S. Häfliger, Ch. Niedermayer, K. Mattenberger, S. Bubenhofer, and B. Batlogg, Phys. Rev. Lett. $100026407(2008)$.

10 D. J. P. Morris et al., arXiv:0803.1312 v2

11 F. C. Chou et al., arXiv:0709.0085 1

12 L. Balicas, Y. J. Jo, G. J. Shu, F. C. Chou, P. A. Lee, Phys. Rev. Lett. 100, 126405(2008).

13 Woei Wu Pai, S. H. Huang, Ying S. Meng, Y. C. Chao, C. H. Lin, H. L. Liu, F. C. Chou, arXiv:0805.0475 1

14 Sen Zhou and Ziqiang Wang, Phys. Rev. Lett. 98, 226402(2007).

15 C. A. Marianetti and G. Kotliar, Phys. Rev. Lett. 98, 176405(2007).

16 Ting-Pong Choy, Dimitrios Galanakis, and Philip Phillips, Phys. Rev. B 75, 073103(2007).

17 T. Wu, D. F. Fang, G. Y. Wang, L. Zhao, G. Wu, X. G. Luo, C. H. Wang and X. H. Chen, Phys. Rev. B 76, 024403(2007).

18 Y. G. Shi, Y. L. Liu, H. X. Yang, C. J. Nie, R. Jin, and J. Q. Li, Phys. Rev. B 70, 052502(2004).

19 M. N. Iliev, A. P. Litvinchuk, R. L. Meng, Y. Y. Sun, J. Cmaidalka, and C. W. Chu, Physica C 402, 239(2004).

20 P. Lemmens, V. Gnezdilov, N. N. Kovaleva, K. Y. Choi, H. Sakurai, E. Takayama-Muromachi, K. Takada, T. Sasaki, F. C. Chou, D. P. Chen, C. T. Lin, and B. Keimer, J. Phys.: Condens. Matter 16, S857(2004).

21 J. F. Qu, W. Wang, Y. Chen, G. Li, and X. G. Li, Phys. Rev. B 73, 092518(2006).

22 Qingming Zhang, Ming An, Shikui Yuan, Yong Wu, Dong Wu, Jianlin Luo, Nanlin Wang, Wei Bao, and Yening Wang, Phys. Rev. B 77, 045110(2008).

23 P. Lemmens, K. Y. Choi, V. Gnezdilov, E. Ya. Sherman, D. P. Chen, C. T. Lin, F. C. Chou, and B. Keimer, Phys. Rev. Lett. 96, 167204(2006).

24 A. Donkov, M. M. Korshunov, I. Eremin, P. Lemmens, V. Gnezdilov, F. C. Chou, and C. T. Lin, Phys. Rev. B 77,
100504(R)(2008).

25 C. H. Wang, X. H. Chen, T. Wu, X. G. Luo, G. Y. Wang, and J. L. Luo, Phys. Rev. Lett. 96, 216401(2006).

26 J. Bobroff, G. Lang, H. Alloul, N. Blanchard, and G. Collin, Phys. Rev. Lett. 96, 107201(2006).

27 D. N. Argyriou, O. Prokhnenko, K. Kiefer, and C. J. Milne, arXiv:0709.1038(2007).

28 F. L. Ning, S. M. Golin, K. Ahilan, T. Imai, G.J. Shu and F. C. Chou, arXiv:0711.4023(2007).

29 G. Garbarino, M. Monteverde, M. Nunez Regueiro, C. Acha, M. L. Foo, R. J. Cava, arXiv:0710.4341 1 